\title{
THE STRUCTURE FUNCTIONS AND LOW $X$ WORKING GROUP SUMMARY
}

\author{
V. CHEKELIAN* (SHEKELYAN), C. GWENLAN** AND R.S. THORNE*** \\ * Max Planck Institute for Physics, Munich, Germany \\ ** University of Oxford, UK \\ *** University College London, UK
}

The structure functions and low $x$ working group summary of DIS06.

\section{Recent results from HERA}

We begin by discussing new results from HERA. In autumn 2003 the accelerator started the second phase of its ep collider programme (HERA-II). The $e^{+} p$ and $e^{-} p$ data collected by the H1 and ZEUS experiments since then, were taken with longitudinally polarised positron and electron beams for the first time. Recent results from HERA-II, related mostly to the exploration of this new feature of the collider, are discussed below. The other HERA result presented was an update of the high- $x$ measurement of the NC cross sections by the ZEUS collaboration ${ }^{1}$. In a discussion session devoted to the longitudinal structure function, $F_{L}$, it was highlighted that as a test of different theoretical models a measurement of $F_{L}\left(x, Q^{2}\right)$ over as wide a range of $x$ and $Q^{2}$ as possible would be very useful ${ }^{2}$, and both H1 and ZEUS expressed a strong interest in running HERA at a low proton energy for about three months, which would allow a direct $F_{L}$ measurement ${ }^{3}$. The experimental and theoretical status of the charm and beauty contributions to $F_{2}$ are summarised in the Heavy Flavour working group summary.

Measurements of charged current (CC) deep inelastic scattering (DIS) with polarised leptons on protons allow tests of the $\mathrm{V}-\mathrm{A}$ structure of $\mathrm{CC}$ interactions to be extended into the high- $Q^{2}$ regime. The polarisation dependence of the CC cross sections is fixed within the Standard Model (SM). Specifically, the SM predicts, from the absence of right handed charged currents, that the $\mathrm{CC} e^{+} p\left(e^{-} p\right)$ cross section is proportional to the fraction of right handed positrons (left handed electrons) in the beam:

$$
\sigma_{\mathrm{CC}}^{e^{ \pm} p}\left(\mathcal{P}_{e}\right)=\left(1 \pm \mathcal{P}_{e}\right) \sigma_{\mathrm{CC}}^{e^{ \pm} p}\left(\mathcal{P}_{e}=0\right)
$$

where the longitudinal polarisation $\mathcal{P}_{e}$ is equal to $\left(N_{R}-N_{L}\right) /\left(N_{R}+N_{L}\right)$ with $N_{R}\left(N_{L}\right)$ being the number of right (left) handed leptons in the beam. $\mathrm{H} 1$ and ZEUS published ${ }^{4}$ the first measurements of the $\mathrm{CC} e^{+} p$ total cross sections for positive and negative longitudinal polarisation, and presented ${ }^{5}$ 


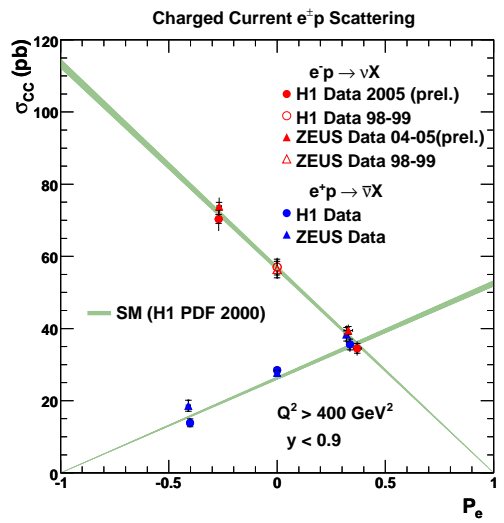

Figure 1. $\quad \mathrm{CC} e^{+} p$ and $e^{-} p$ cross sections versus $\mathcal{P}_{e}$ compared to the SM prediction.

the $\mathrm{CC} e^{-} p$ total cross sections. The value of the polarisation was typically between $30 \%$ and $40 \%$. The measured integrated CC cross sections, $\sigma_{\mathrm{CC}}^{e^{ \pm}}$, quoted in the range $Q^{2}>400 \mathrm{GeV}^{2}$ and $y<0.9$, are shown in Fig. 1 . The measurement of the unpolarised total cross section, in the same phase space, based on data collected until 2000 (HERA-I) is also shown. The measurements are compared to SM predictions based on the H1 PDF 2000 parametrisation $^{6}$. The measurements agree with SM predictions and exhibit the expected linear dependence as a function of the polarisation. Linear fits provide a good description of the data and their extrapolation to the point $\mathcal{P}_{e}=-1\left(\mathcal{P}_{e}=1\right)$ yield a fully left (right) handed CC cross section for $e^{+} p\left(e^{-} p\right)$ interactions which is consistent with the vanishing SM prediction. The corresponding upper limits on the total CC cross sections exclude the existence of charged currents involving right handed fermions mediated by a boson of mass below $180-208 \mathrm{GeV}$ at $95 \%$ confidence level, assuming SM couplings and a massless right handed $\nu_{e}$.

The NC single differential cross sections $\mathrm{d} \sigma / \mathrm{d} Q^{2}$ have been measured at HERA-II for $e^{+} p$ and $e^{-} p$ scattering with positively and negatively polarised lepton beams ${ }^{7}$. The SM predicts a difference in the cross section for leptons with different helicity states arising from the chiral structure of the neutral electroweak exchange. In Fig. 2 the ratio of cross sections for positively and negatively polarised lepton beams is shown separately for $e^{+} p$ (left plots) and $e^{-} p$ (right plots) scattering data. In both cases the ratio is found to be consistent, within experimental uncertainties, with unity at low $Q^{2}$, indicating little dependence of the cross section on beam polarisation. The normalisation uncertainties of the measurements are not included in the errors bars, but are indicated by the dashed lines on the upper figures. At higher $Q^{2}$, the data have a tendency to deviate from unity. For $e^{+} p$ scattering the data indicate that right-handed positrons yield a larger cross 

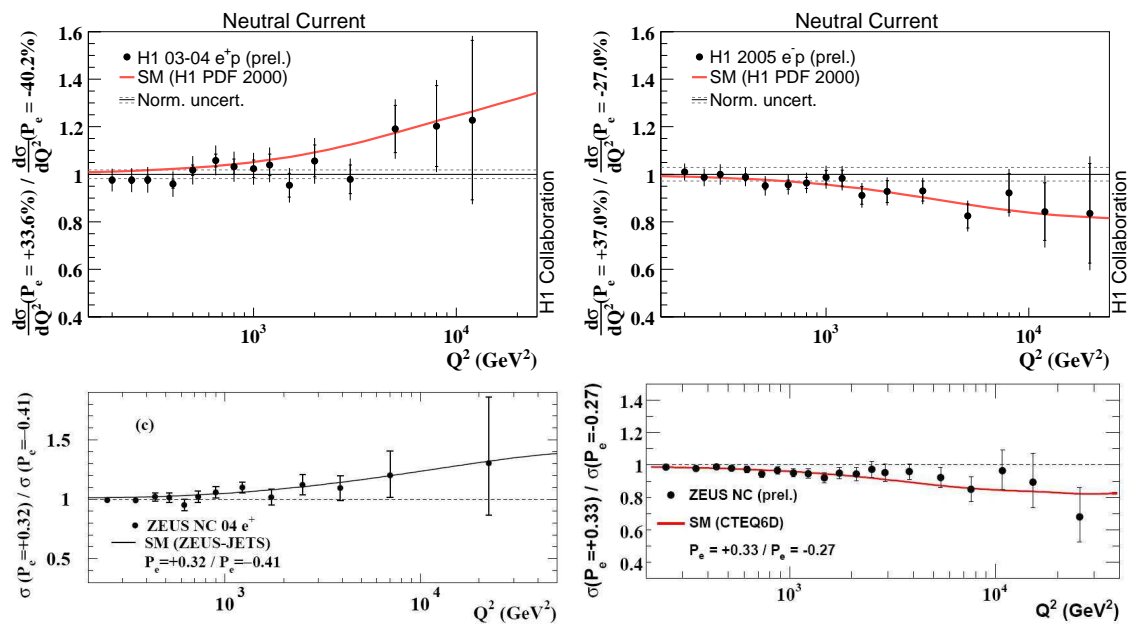

Figure 2. The ratios of the $\mathrm{NC}$ cross sections for leptons with positive and negative polarisations at HERA-II measured by H1 (top) and ZEUS (bottom) as function of $Q^{2}$, for $e^{+} p$ (left) and $e^{-} p$ (right). Solid lines correspond to the SM expectation.

section than left-handed ones, whereas for $e^{-} p$ scattering the data show the opposite behaviour. This is consistent with the SM expectation shown as the solid curve in Fig. 2.

At HERA, the NC interactions at high $Q^{2}$ receive contributions from $\gamma Z$ interference and $Z^{0}$ exchange. Thus, the NC data can be used to extract the weak couplings of the light $u$ and $d$ quarks to the $Z^{0}$ boson. The $\mathrm{CC}$ cross section data help disentangle the up and down quark distributions and allow a determination of a propagator mass $M_{\text {prop }}$ from the $Q^{2}$ dependence of the cross sections. $M_{\text {prop }}$ measured at HERA in the space-like region can be compared with direct $W$ boson mass measurements obtained in the time-like region by the Tevatron and LEP experiments. Combined electroweak and QCD fits at HERA have been performed ${ }^{8,9}$, taking into account the correlation between the electroweak parameters and the parton distribution functions (PDFs). The ZEUS collaboration extend their NLO QCD fit ${ }^{10}$ to inclusive NC and CC data, inclusive jet data in DIS and dijets in photoproduction, to determine simultaneously the PDFs and electroweak parameters. This fit also includes, for the first time, the $e^{ \pm} p$ polarised NC and CC double-differential HERA-II cross sections. The resulting PDFs are very similar to those obtained from the fit to HERA-I data, with slightly improved uncertainties on the $u$-quark at high $x$, due to additional data. In this analysis, the fits have been performed by fixing either the $u$ or the $d$ quark couplings to their SM values (fits $v_{d^{-}} a_{d^{-}}$PDF and $\left.v_{u}-a_{u}-\mathrm{PDF}\right)$. The resulting one-sigma contours are shown in Fig. 3. 

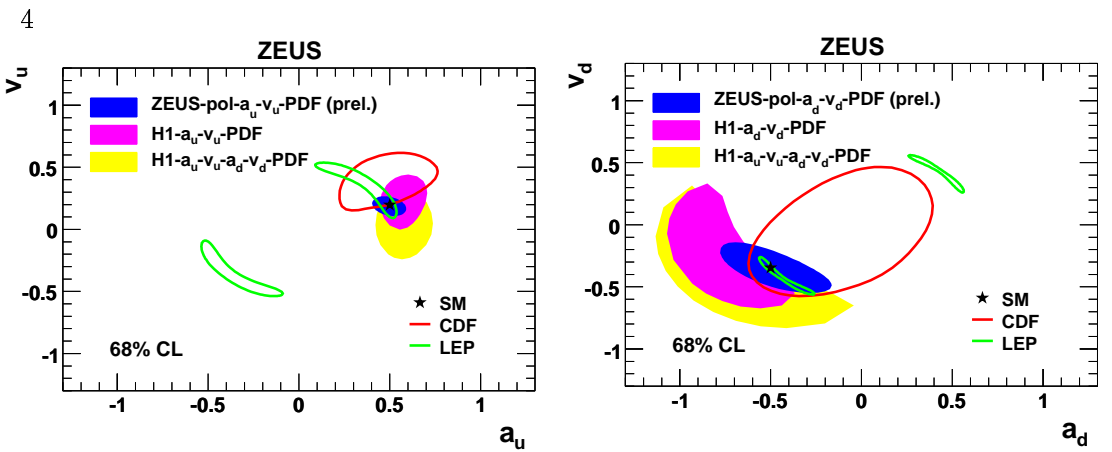

Figure 3. Contours of the $68 \%$ confidence level (CL) on the weak neutral current couplings of $u$ (left plot) and $d$ (right plot) quarks to the $Z^{0}$ boson.

The results are consistent with the electroweak Standard Model and the precision is better for the $u$ quark as expected. Comparison to the H1 fits to unpolarised HERA-I data shows that, while the uncertainty on the axialvector couplings stays about the same, the precision of the determination of the vector couplings is improved by a factor of 2-3 due to additional sensitivity of the polarised NC data. Figure 3 also shows the results of the $\mathrm{H} 1$ fits in which the vector and axial-vector couplings of $u$ and $d$ quarks are fitted simultaneously (fits $v_{u}-a_{u}-v_{d^{-}} a_{d^{-}} \mathrm{PDF}$ ) and similar results obtained recently by the CDF experiment and at LEP. The HERA determinations have comparable precision to that from the Tevatron and resolve any sign ambiguity and the ambiguities between $v_{u}$ and $a_{u}$ of the determinations based on observables measured at the $Z^{0}$ resonance. Exploiting the $Q^{2}$ dependence of the charged current data, the propagator mass has been measured to be $M_{\text {prop }}=82.8 \pm 1.5 \pm 1.3 \mathrm{GeV}$, which is in agreement with the direct measurements of the $W$ boson mass.

The amount of data collected at HERA-II is already greater than that of HERA-I. In particular, a significant increase of integrated luminosity is achieved in the $e^{-} p$ mode, from $\approx 15 \mathrm{pb}^{-1}$ to more than $100 \mathrm{pb}^{-1}$. At HERA-I the statistics of the $e^{-} p$ data was a limiting factor for the precision of the $x F_{3}$ determination. Profiting from the enlarged statistics and reduced systematic uncertainties, the previous measurement of $x F_{3}$ has been updated ${ }^{7}$ using HERA-II 2003-2005 $e^{ \pm} p$ NC cross section data at high $Q^{2}$. Fig. 4 (left top) shows the comparison of the unpolarised $e^{-} p$ and $e^{+} p$ HERA-II $\mathrm{H} 1$ data for three different $Q^{2}$ values. At high $Q^{2}$, the NC cross section in $e^{-} p$ scattering is significantly larger than that in $e^{+} p$ scattering due to the different sign of the $x F_{3}$ contribution to the cross section for different leptons polarities. $x F_{3}$ determined by ZEUS from the difference of $e^{-} p$ and $e^{+} p$ cross sections is shown in Fig. 4 (right). The dominant contribution to $x F_{3}$ arises from the $\gamma Z$ interference, which 

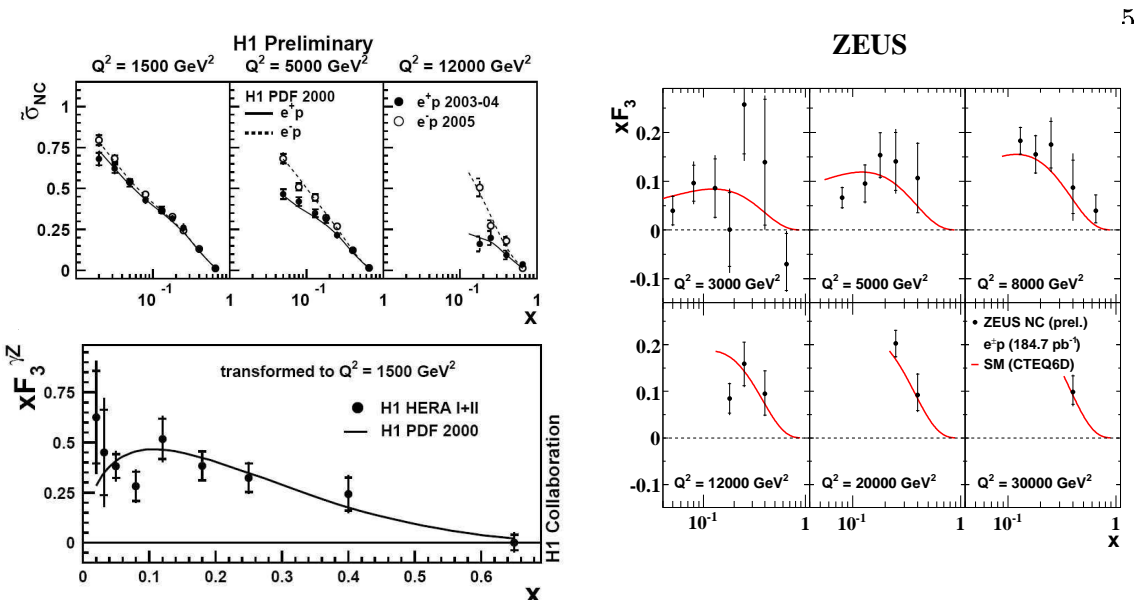

Figure 4. The NC reduced cross sections $\tilde{\sigma}_{N C}^{ \pm}\left(x, Q^{2}\right)$ measured by H1 at HERA-II (left top), the structure functions $x F_{3}$ from ZEUS (right) and the averaged H1 structure function $x F_{3}^{\gamma Z}$ for $Q^{2}=1500 \mathrm{GeV}^{2}$ (left bottom) compared to QCD expectations.

allows $x F_{3}^{\gamma Z}$ to be extracted according to $x F_{3}^{\gamma Z} \simeq-x F_{3}\left(Q^{2}+M_{Z}^{2}\right) /\left(a_{e} \kappa Q^{2}\right)$ by neglecting the pure $Z$ exchange contribution, which is suppressed by the small vector coupling $v_{e}$. Here, $\kappa^{-1}=4 M_{W}^{2} / M_{Z}^{2}\left(1-M_{W}^{2} / M_{Z}^{2}\right), M_{Z}$, $M_{W}$ are the vector boson masses and $a_{e}$ is the axial-vector coupling of the electron. This structure function is non-singlet and has little dependence on $Q^{2}$. The measured $x F_{3}^{\gamma Z}$ at different $Q^{2}$ values can thus be averaged taking into account the small $Q^{2}$ dependence. The averaged $x F_{3}^{\gamma Z}$, determined by H1 for $Q^{2}=1500 \mathrm{GeV}^{2}$, is shown in Fig. 4 (left bottom) compared to the QCD fit result. The structure function $x F_{3}^{\gamma Z}$ determines both the shape and magnitude of the valence quark distributions independently of the sea quark distributions.

\section{FNAL, JLAB, RHIC and new methods for data analysis}

In this section, we summarise a number of new results from experiments at Fermilab, JLab and RHIC, and discuss several new approaches for determining PDFs and their uncertainties.

Recently, the importance of reducing the proton PDF uncertainties, especially at high $x$, has become increasingly apparent, both for precision SM studies and new physics searches at hadron colliders. The CDF and D0 collaborations presented a number of QCD and electroweak measurements that could provide additional constraints on the $\mathrm{PDFs}^{11,12}$. Of particular importance is the, poorly known, high- $x$ gluon distribution, which can be constrained using hadron collider jet data. CDF and D0 presented new measurements of inclusive jets ${ }^{11,12}$ in $p \bar{p}$ collisions. The data agree well with NLO QCD predictions over the measured $E_{T}$ range, which extends to 




Figure 5. The distribution of $x s^{-}$at $Q^{2}=16 \mathrm{GeV}^{2}$.

$\sim 600-700 \mathrm{GeV}$. The precision of the data, which is limited by the jet energy scale uncertainty, is at a level where these measurements should provide significant additional constraints on the high- $x$ gluon. In particular, in the most forward region measured by CDF $(1.6<|y|<2.1)$ experimental uncertainties are smaller than those from the PDFs. Note that measurements over a wide range of rapidity are important to discriminate between PDF effects and new physics signals. CDF and D0 also presented a number of other results ${ }^{11,12}$, including measurements of the forward-backward charged lepton asymmetry from $W^{ \pm} \rightarrow l^{ \pm} \nu$ decays, which constrains the $d / u$ ratio, a new measurement of the forward $W \rightarrow e \nu$ cross section and studies of $Z \rightarrow \mu \mu, Z \rightarrow \tau_{e} \tau_{h}, Z+$ jets and dijet azimuthal decorrelations.

NLO QCD calculations of jet cross sections are CPU intensive, rendering their use in QCD fits challenging. Global fit groups ${ }^{13,14}$ use LO cross sections $+k$-factors to approximate the NLO result. At this workshop, an approach was presented ${ }^{15}$, in which an NLO QCD program is used to calculate a grid of weights in $\left(x, Q^{2}\right)$, which can be convoluted with any PDF to give fast NLO predictions ${ }^{\mathrm{a}}$. The accuracy of the grid-computed cross sections is improved, without significant cost to CPU, by using coordinate transformations and high order interpolation between grid points. This technique will allow the rigorous inclusion of HERA, Tevatron and LHC jet data in future QCD fits.

$\mathrm{NuTeV}$ presented the final measurement of the difference between the strange and antistrange quark distributions ${ }^{17}$. This is a topic of particular interest, since it has been speculated ${ }^{18}$ that a non-zero difference - or strange asymmetry - could explain the almost $3 \sigma$ difference between the $\mathrm{NuTeV} \sin ^{2} \theta_{W}$ result ${ }^{19}$ and the world average. $\mathrm{NuTeV}$ have performed the first complete NLO QCD analysis of CC $\nu N$ and $\bar{\nu} N$ scattering, with two oppositely charged muons in the final state, giving direct access to the

$\overline{{ }^{a} \text { A similar grid technique was also }}$ presented $^{16}$ in the Hadronic Final States session. 
strange quark content of the nucleon. Figure 5 shows the distribution of $x s^{-}(x)=x s(x)-x \bar{s}(x)$ extracted from the fit, which is positive at moderate $x$, such that $S^{-} \equiv \int_{0}^{1} x s^{-}(x) d x=+0.00196 \pm 0.00143$. NuTeV are now updating their $\sin ^{2} \theta_{W}$ result, which originally assumed $S^{-}=0$, in light of this result, and also taking into account other recent measurements, such as the BNL $K_{e 3}^{+}$measurement ${ }^{20}$. Note also, that the data prefer an asymmetry which forces $s^{-}(x)$ negative at low values of $x_{0} \lesssim 0.004$, in contrast to some theoretical models, which suggest larger values of $x_{0}$.

The E03-103 experiment at JLab have performed a new measurement of the EMC effect in light nuclei ${ }^{21}$. The characteristic $x$-dependence of the ratio $\sigma_{A} / \sigma_{d}$ (i.e. the so-called "EMC effect"), has been well studied. While broad features can be explained, no single model has successfully described the effect over all $x$. To date, much of the experimental effort has been concentrated on heavy nuclei $(A>4)$. However, calculations of the EMC effect in ${ }^{3} \mathrm{He}$ and ${ }^{4} \mathrm{He}$ predict large differences in both magnitude and $x$ dependence. E03-103 have measured inclusive electron scattering, using a $5.8 \mathrm{GeV}$ beam, providing the first measurement of the EMC effect in ${ }^{3} \mathrm{He}$ for $x>0.4$, and improving upon existing measurements ${ }^{22}$ of ${ }^{4} \mathrm{He}$. The results indicate that the effect in ${ }^{4} \mathrm{He}$ is similar to that in carbon and the effect in ${ }^{3} \mathrm{He}$ could be larger than expected, although the strength of the latter conclusion is limited by large uncertainties in proton excess corrections.

JLab also presented results on the phenomenon of parton-hadron duality in semi-inclusive scattering. Duality, for example the fact that the average behaviour of nuclear resonances exhibits the scaling behaviour expected in pQCD, is well established in inclusive scattering, but has yet to be studied to the same extent in semi-inclusive data. The merit of semi-inclusive processes lies in the ability to identify individual quark species in the nucleon by tagging specific mesons in the final state. The E00-108 experiment at JLab presented ${ }^{23}$ preliminary measurements of semi-inclusive pion electroproduction, $e N \rightarrow e^{\prime} \pi^{ \pm} N$, using a $5.5 \mathrm{GeV}$ electron beam on proton and deuteron targets, for $1.8<Q^{2}<6.0 \mathrm{GeV}^{2}, 0.3 \leq x \leq 0.55$, and elasticities, $z=E_{\pi} / \nu$, in the range $0.35-1$. The results indicate that the ratio of the unfavoured to favoured fragmentation functions, $\frac{D^{-}}{D^{+}}=\frac{4-N_{\pi}^{+} / N_{\pi}^{-}}{4 N_{\pi}^{+} / N_{\pi}^{-}-1}$, shows no dependence on $x$ and a smooth slope as a function of $z$, in accordance with the expectations of the onset of duality. It was argued that these data are also consistent with the idea that pion electroproduction is related to low energy factorisation. If so, future semi-inclusive measurements could give excellent access to the flavour structure of the nucleon. 

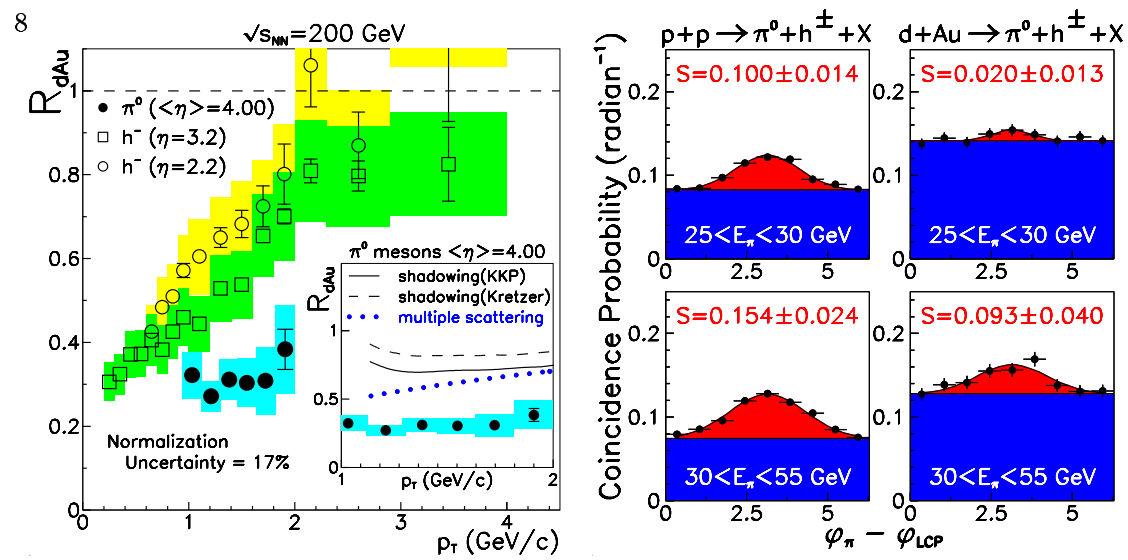

Figure 6. Left: nuclear modification factor, $R_{\mathrm{dAu}}=\frac{1}{2 \times 197} \sigma_{\mathrm{d}+\mathrm{Au}} / \sigma_{\mathrm{p}+\mathrm{p}}$, as a function of $p_{\mathrm{T}}$ for $\pi^{0}$ mesons at $\langle\eta\rangle=4.00$ and charged hadrons at smaller pseudorapidities. Error bars are statistical, while the shaded boxes are point-to-point systematics. Right: azimuthal correlations between forward $\pi^{0}$ and charged hadrons at mid-rapidity.

The STAR collaboration have searched for parton saturation effects at $\mathrm{RHIC}^{24}$. The term saturation describes the idea that at very small $x$, the gluon density is so large, that gluons begin to recombine, leading to a slowing of the growth and giving rise to non-linear terms in the evolution equations. The state of saturation is often referred to as the Colour Glass Condensate (CGC). Nuclear environments, such as RHIC, are ideal places to search for signs of saturation, due to the $A^{1 / 3}$ enhancement of the parton densities in nuclei, relative to the proton. STAR presented results on forward $\pi^{0}$ production in $\mathrm{p}+\mathrm{p}$ and $\mathrm{d}+\mathrm{Au}$ collisions, in the range $3.0 \lesssim \eta \lesssim 4.2$, at $\sqrt{s}=200 \mathrm{GeV}$. Measurements at forward rapidities ensure that low values of gluon- $x$ are probed. The $\mathrm{p}+\mathrm{p}$ yields generally agree with NLO pQCD predictions, while the $\mathrm{d}+\mathrm{Au}$ yield is suppressed at forward rapidities, as shown in Fig. 6 (left). It was argued that the $p_{\mathrm{T}}$ dependence of the $\mathrm{d}+\mathrm{Au}$ yield is consistent with a model treating the $\mathrm{Au}$ nucleus as a CGC for forward pion production ${ }^{24}$. The azimuthal correlations of the forward $\pi^{0}$ with charged hadrons at mid-rapidities (Fig. 6 (right)) show a recoil peak in $\mathrm{p}+\mathrm{p}$ that is suppressed in $\mathrm{d}+\mathrm{Au}$ collisions at low $E_{\pi}$; in qualitative agreement with a gluon saturation picture of the $\mathrm{Au}$ nucleus. Note, however, that the PHENIX experiment at RHIC do not see significant differences in the $\mathrm{p}+\mathrm{p}$ and $\mathrm{d}+\mathrm{Au}$ azimuthal correlations ${ }^{25}$. STAR will soon make further measurements, utilising a new forward meson spectrometer, that could elucidate the source of the observed suppression.

Several presentations were made in which alternative approaches to PDF determination were explored. The NNPDF collaboration presented the latest results on a neural network (NN) approach to PDF fitting ${ }^{26}$. This 
approach attempts to avoid any bias introduced from a choice of functional form for the PDFs and should provide more faithful estimates of the PDF uncertainties. The NNPDF best-fit of the non-singlet structure function, $F_{2}^{N S}\left(x, Q^{2}\right)=F_{2}^{p}\left(x, Q^{2}\right)-F_{2}^{d}\left(x, Q^{2}\right)$, agrees with the predictions of other PDF sets, and gives a better description at large $x$, as well as larger error bands in the region where there is no data. Work is now in progress to construct a full set of PDFs. A related approach was presented by the SOMPDF group ${ }^{27}$. This technique is based on a specific class of NN, called the Self Organising Map (SOM). It was argued that SOMs allow better control of systematic bias, by replacing the fully automated procedure of standard NNs, with an interactive fitting procedure. Finally, the prospects for determining PDFs and their uncertainties using a Bayesian statistical approach, was discussed ${ }^{28}$. This work is at an early stage, but it will be extremely interesting to see the results when they become available.

\section{Theory}

The main topics were updates in determinations of proton and nuclear partons distributions and the ever increasing sophistication (complication?) of theory. The former included the implementation of new heavy flavour prescriptions and/or NNLO corrections and new data in fits. The latter included recent developments in small- $x$ resummations, with hopefully the beginning of detailed understanding and phenomenology. Matters are getting more involved in the very small $x$ region with non-linear effects, and the saturation scale seems to be becoming ever smaller.

Starting with fits to partons distributions, Guffanti presented a NNLO analysis of non-singlet parton distributions ${ }^{29} u_{v}\left(x, Q^{2}\right)$ and $d_{v}\left(x, Q^{2}\right)$ by fitting to $F_{2}^{p, d}\left(, x, Q^{2}\right)$, for $x>0.3$ and $F_{2}^{p}-F_{2}^{d}$. The result is $\alpha_{S}\left(M_{Z}^{2}\right)=$ $0.1134 \pm 0.0020$, in good agreement with other NNLO fits to only structure function data. Alekhin made his fits more global ${ }^{30}$ by including E605 DrellYan data ${ }^{31}$ and E866 Drell-Yan ratio data ${ }^{32}$. The fit has no problems with this data, and it improves the accuracy on the high- $x$ sea and gives his first real constraint on $\bar{u}-\bar{d}$. In fact using $\Delta \chi^{2}=1$ the sea uncertainties are very small. The theoretical input to the DIS fit is the massless NNLO QCD corrections for the light quarks and gluons (splitting ${ }^{33}$ and coefficient functions ${ }^{34,35}$; account of the heavy quarks up to $O\left(\alpha_{S}^{2}\right)^{36}$ in FFNS; account of the target-mass corrections, Fermi-motion in deuterium, and twist 4 terms; and the massless $O\left(\alpha_{S}^{3}\right)$ corrections ${ }^{37}$ to the coefficient functions. There is disagreement with the definition of NNLO regarding heavy quarks

- this is conventionally only NLO corrections in the FFNS scheme. The last 
step is only part of a full $\mathrm{N}^{3} \mathrm{LO}$ correction - lacking the splitting functions and not necessarily indicative except at high $x$. Reasonable stability is claimed down to $Q^{2}=0.5 \mathrm{GeV}^{2}$ for $0.06 \leq x \leq 0.12$ - perhaps possible since most corrections are small in this region.

There are some issues with new data in the fits. Most interesting was the $\mathrm{NuTeV}$ structure function data ${ }^{38}$ which is larger than the similar CCFR data $^{39}$ at high $x$ and is useful for flavour separation. Comparison to this data relies on nuclear corrections. A determination of these was reported by Kumano $^{40}$. CTEQ find these $\mathrm{NuTeV}$ data difficult to fit ${ }^{41}$, whilst MRST only obtain a good fit by severely reducing the nuclear correction ${ }^{42}$, implying that this is different for neutrinos than for charged leptons. The important information in the data is for the region $x<0.3$ which is not too sensitive to the nuclear corrections, but the problem caused much interest. Recent CHORUS data ${ }^{43}$ are more similar to CCFR data. There are also some changes in global fit procedures. $\mathrm{CTEQ}^{41}$ include all HERA data and fit directly to cross-sections for first time which requires $F_{L}\left(x, Q^{2}\right)$ at high $y$. They also implement a new heavy flavour prescription ${ }^{44}$ (see the Heavy Flavour session summary). It was noted that the new fit overshoots the high- $y$ data, with the match between theory and data requiring the use of the photoproduction background systematic error at more than $2 \sigma$, i.e. use of the systematic errors can remove the high- $y F_{L}\left(x, Q^{2}\right)$ turnover. MRST implement a full NNLO VFNS ${ }^{45}$ and use the Drell-Yan cross-sections $^{35}$ for the first time, leading to (provisional) full NNLO partons with uncertainties ${ }^{42}$. The improvement in the charm procedure affects the gluon compared to the approximate MRST2004 NNLO fit ${ }^{14}$. The quality of the fit at NNLO is consistently better than NLO, but not for Drell-Yan data. There is a definite tendency for $\alpha_{S}\left(M_{Z}^{2}\right)$ to go down with all the changes - at NLO $\alpha_{S}\left(M_{Z}^{2}\right)=0.121$ and at NNLO $\alpha_{S}\left(M_{Z}^{2}\right)=0.119$.

There were presentations on various ways to include small- $x$ resummations from the BFKL equation on top of the fixed order expansion. White showed a resummation of $\ln (1 / x)$ terms along with running coupling corrections ${ }^{46}$, which produces mainly analytic results with small numerical corrections. The procedure includes the quark-gluon splitting $P_{q g}$ and the full implementation of a heavy flavour $\mathrm{VFNS}^{47}$ and gives full LO resummed partons. Colferai outlined an approach which is also applicable for processes with two hard scales. It too includes the running coupling but also resummation of collinear singularities:

$$
\int d k^{2}\left(k^{2}\right)^{-\gamma-1} K^{n}\left(k^{2}\right)=\chi^{n}(\gamma), \quad \chi^{n}(\gamma) \sim 1 / \gamma^{2 n+1}, 1 /(1-\gamma)^{2 n+1}
$$


The evolution variable is $s /\left(Q Q_{0}\right)$ with conjugate variable $N$. Consideration of changes of evolution variable to $s / Q^{2}$ (DIS) and $s / Q_{0}^{2}$ lead to the resummation ${ }^{48} \chi_{N}^{n}(\gamma) \sim 1 /(\gamma+N / 2)^{n+1}, 1 /(1-\gamma+N / 2)^{n+1}$. The natural calculations are in DIS scheme with the incoming gluon offshell $-k^{2}=Q_{0}^{2} \neq 0$. Colferai looked at the transformation to the $\overline{\mathrm{MS}}$ scheme ${ }^{49}$, showing that the effect is not large. Forte presented an approach based on: duality - one has $\chi\left(\gamma\left(N, \alpha_{S}\right), \alpha_{S}\right)=N, \gamma\left(\chi\left(\alpha_{S}, M\right), \alpha_{S}\right)=N$ i.e. the $Q^{2}$ evolution and $x$ evolution are dual ${ }^{50}$ (perhaps not the most important issue); explicit imposition of momentum conservation; inclusion of the running of the coupling ${ }^{51}$; and symmetrisation, i.e. letting $1 / M^{n} \rightarrow 1 /(M+N / 2)^{n}, 1 /(1-M)^{n} \rightarrow 1 /(1-M+N / 2)^{n}$. Overall this leads to a resummed $P_{g g}\left(x, Q^{2}\right)^{52}$. These methods are all rather different in details, but now have many similarities, and produce similar results for $P_{g g}$ at NLO in the resummation, with a dip below the LO splitting function for $x \sim 0.001$, and a slow growth not setting in until $x \sim 10^{-5}$. White also examines phenomenology, at LO in the resummation only - the impact factor required for $P_{q g}$ is not yet known at NLO. The analysis gives a better fit ${ }^{47}$ than NLO-in- $\alpha_{S}$ in terms of $d F_{2}\left(x, Q^{2}\right) / d \ln Q^{2}$, but the enhancement of the evolution is too great at small $x$ and the resulting gluon and $F_{L}\left(x, Q^{2}\right)$ are too small at moderate $x$, suggesting that we need the full NLO generalization. Colferai ${ }^{49}$ and White ${ }^{53}$ both examine the improvement to $P_{q g}$. The two approaches are qualitatively similar but contain different higher order information: White estimates NLO corrections to the impact factor ${ }^{54}$; Colferai has a resummation of $P_{g g}$ beyond NLO via the collinear resummation. Both suggest the effects of the NLO resummation beyond fixed order in the quark sector are small but significant, as seen in Fig.7.
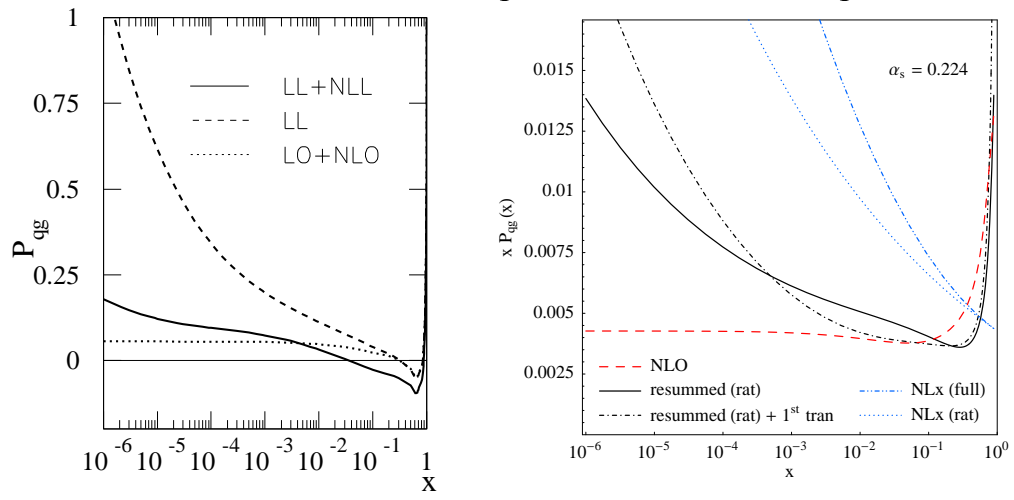

Figure 7. The splitting function $P_{q g}$ in two approaches including small $x$ resummations.

There was an update on non-linear corrections at very small $x$, with 
various discussions on how to include the saturation corrections, and more. Soyez considered the nonlinear evolution equation in rapidity $Y$ extended to include fluctuations as well as recombination ${ }^{55}$, i.e. the equation is

$\partial_{Y}\left\langle T^{(n)}\right\rangle=\bar{\alpha} M_{B F K L} \otimes\left\langle T^{(n)}\right\rangle-\bar{\alpha} M_{B F K L} \otimes\left\langle T^{(n+1)}\right\rangle+\bar{\alpha} \alpha_{S}^{2} K \otimes\left\langle T^{(n-1)}\right\rangle$,

where $T^{m}$ is the amplitude for $m$ dipole scattering. Hatta demonstrated the origin of the fluctuations via a formal derivation of a Bremsstrahlung Hamiltonian $^{56}$ which gives the evolution of $n$-dipole densities. Soyez showed that the inclusion of the fluctuations leads to a dispersion about the saturation scale ${ }^{57}$. One can be sensitive to saturation effects when nominally far away from the saturation scale, or conversely, when naively near this scale, fluctuations to even lower $x$ have no effect since saturation is already reached, while those to higher scales reduce saturation effects. The net effect is to move the onset of saturation in the dipole cross-section considerably downwards, as seen in Fig.8, which compares the dipole cross-section with and without fluctuations. Kutak modifies the LO BFKL equation to include a non-linear recombination term ${ }^{58}$ and also investigates impact parameter dependence ${ }^{59}$. Moreover, high- $x$ effects in the gluon evolution are accounted for (though not in the gluon-quark impact factor, i.e. $P_{q g}$ ). The impact parameter dependence reduces the effect of the nonlinear term and lowers the saturation scale in $Q^{2}$ for fixed $x$, i.e. peripheral collisions see smaller densities. The conclusion from some rough phenomenology is that we "hardly see the effect of saturation" at HERA.



Figure 8. Comparison of the dipole cross-section from a fit with only saturation corrections (solid) and one which also allows for fluctuations (dots).

To summarise, there is little agreement in global fit analyses. Not everyone wants to go to NNLO, and not everyone agrees how to do it in detail. However, it seems we are now at the stage where NNLO parton analyses are essentially complete and reliable and should be done. They work a little better than NLO in general. There are rather similar results coming from 
all groups working on small- $x$ resummations to be used in conjunction with fixed order calculations, though differences in procedures. The effect of the resummations is moderate until very small $x$. Empirically, their inclusion can improve the fit a little over NLO. There is progress in nonlinear small- $x$ equations, e.g. fluctuations, which always seems to be pushing the saturation scale lower. It would be good if this approach could match on to higher $x$ better - at present it is usually confined to some unknown small $x$ region, and is missing higher $x$ corrections. Overall there is lots of improvement in how to calculate using different techniques. However, there is not enough idea yet where each approach is applicable/needed. We still need better (real) phenomenology and, of course, more useful data.

\section{References}

1. Allen Caldwell (ZEUS Collab.), these proceedings.

2. R.S. Thorne, the proceedings of "New Trends in HERA Physics 2005" Ringberg Castle, Tegernsee, October 2005, p. 359. hep-ph/0511351.

3. Daniel Kollar (ZEUS Collab.), these proceedings; Max Klein (H1 Collab.), these proceedings; Allen Caldwell, plenary talk, these proceedings.

4. H1 Collab., A. Aktas et al., Phys. Lett. B 634, 173 (2006); ZEUS Collab., S. Chekanov et al., DESY-06-015, accepted by Phys. Lett. B.

5. Biljana Antunovic (H1 Collab.), these proceedings; Hiroshi Kaji (ZEUS Collab.), these proceedings.

6. H1 Collab., C. Adloff et al., Eur. Phys. J. C30, 1 (2003).

7. Andrei Nikiforov (H1 Collab.), these proceedings; Umer Noor (ZEUS Collab.), these proceedings.

8. H1 Collab., A. Aktas et al., Phys. Lett. B 632, 35 (2006).

9. Shima Shimizu (ZEUS Collab.), these proceedings.

10. ZEUS Collab., S. Chekanov et al., Eur. Phys. J. C42, 1 (2005).

11. C. Issever (CDF Collab.), these proceedings.

12. M. Voutilainen (D0 Collab.), these proceedings.

13. J. Pumplin et al., JHEP, 0207, 012 (2002).

14. A.D. Martin et al., Phys. Lett. B604 61 (2004).

15. T. Carli, G. P. Salam, F. Siegert, hep-ph/0510324; D. Clements, these proceedings.

16. M. Wobisch, these proceedings.

17. D. Mason (NuTeV Collab.), these proceedings.

18. S. Davidson et al. JHEP 0202, 037 (2000); NuTeV Collab., G. P. Zeller et al., [Erratum-ibid. D67, 119902 (2003)]; S. Kretzer et al., hep-ph/0312322.

19. NuTeV Collab., G. P. Zeller et al., Phys. Rev. Lett. 88091802 (2002); [Erratum-ibid. 90, 239902 (2003)].

20. E865 Collab., A. Sher et al., Phys. Rev. Lett. 91, 261802 (2003).

21. J. Seely (E03-103 Collab.), these proceedings.

22. E139 Collab., J. Gomez et al., Phys. Rev. D49, 4348 (1994).

23. R. Ent (E00-108 Collab.), these proceedings. 
24. STAR Collab., J. Adams et al., nucl-ex/0602011; C. Gagliardi (STAR Collab.), these proceedings.

25. PHENIX Collab., S. S. Adlet et al., Phys. Rev. Lett. 96, 222301 (2006).

26. NNPDF Collab., A. Piccione et al., hep-ph/0607199; these proceedings.

27. SOMPDF Collab., S. Liuti et al., these proceedings.

28. G. Cowan, these proceedings.

29. J. Blumlein et al., hep-ph/0607200; these proceedings, hep-ph/0606309.

30. S. Alekhin, K. Melnikov and F. Petriello, hep-ph/0606237.

31. E605 Collaboration: G. Moreno et al., Phys. Rev. D43 (1991) 2815.

32. E866 Collaboration: R.S. Towell et al., Phys. Rev. D64 052002 (2001).

33. S. Moch et al., Nucl. Phys. B688 101 (2004); Nucl. Phys. B691 129 (2004).

34. E.B. Zijlstra and W.L. van Neerven, Phys. Lett. B272 127 (1991); ibid. B273 476 (1991); ibid B297 377 (1992); Nucl. Phys. B383 525 (1992).

35. C. Anastasiou et al., Phys. Rev. Lett. 91, 182002 (2003); Phys. Rev. D69, 094008 (2004).

36. E. Laenen et al., Nucl. Phys. B392 162 (1993); B.W. Harris and J. Smith, Nucl. Phys. B452 109 (1995).

37. J.A.M. Vermaseren, A. Vogt and S. Moch, Nucl. Phys. B724, 3 (2005).

38. NuTeV Collab., M. Tzanov et al., Int. J. Mod. Phys. A20, 3759 (2005).

39. CCFR Collaboration: W.G. Seligman et al., Phys. Rev. Lett. 791213 (1997); CCFR Collaboration: U.K. Yang et al., Phys. Rev. Lett. 862742 (2001).

40. S. Kumano, these proceedings; M. Hirai, S. Kumano and T.H. Nagai, Phys. Rev. C70, 044905 (2004).

41. W.K. Tung, these proceedings.

42. R.S. Thorne et. al., hep-ph/0606244.

43. CHORUS Collaboration: G. Onengut et al., Phys. Lett. B632 65 (2006).

44. W.K. Tung et al., J. Phys. G28 983 (2002); S. Kretzer et al., Phys. Rev. D69 114005 (2004).

45. R.S. Thorne, Phys. Rev. D73 054019 (2006).

46. R. S. Thorne, Phys. Lett. B474, 372 (2000); Phys. Rev. D64, 074005 (2001).

47. C.D. White and R.S. Thorne, Phys. Rev. D47, 014002 (2006).

48. G.P. Salam, JHEP 9807, 019 (1998).

49. M. Ciafaloni and D. Colferai, JHEP 0509, 069 (2005); M. Ciafaloni et al., Phys. Lett. B635, 320 (2006).

50. G. Altarelli et al., Nucl. Phys. B575, 313 (2000).

51. G. Altarelli et al., Nucl. Phys. B621, 359 (2002)

52. G. Altarelli et al., Nucl. Phys. B742, 1 (2006)

53. C.D. White, these proceedings, hep-ph/0605321.

54. C. D. White and R. S. Thorne, Eur. Phys. J. C45, 179 (2006).

55. E. Iancu et al., Nucl. Phys. A768, 194 (2006).

56. Y. Hatta et al., Nucl. Phys. A762, 272 (2005).

57. G. Soyez, Phys. Rev. D72, 016007 (2005).

58. K. Kutak and A.M. Stasto, Eur. Phys. J. C41, 343 (2005)

59. K. Kutak, these proceedings. 